\title{
Modular adaptive optics solution for a QKD receiver on a fork mount telescope system
}

E. Fischer, K. Kudielka, A. Brady, A. Kamm, T. Berkefeld, et al.

E. Fischer, K. Kudielka, A. Brady, A. Kamm, T. Berkefeld, R. Ursin, "Modular adaptive optics solution for a QKD receiver on a fork mount telescope system," Proc. SPIE 11852, International Conference on Space Optics ICSO 2020, 118520X (11 June 2021); doi: 10.1117/12.2599216

SPIE Event: International Conference on Space Optics - ICSO 2021, 2021, Online Only 


\section{International Conference on Space Optics-ICSO 2020}

Virtual Conference

30 March-2 April 2021

Edited by Bruno Cugny, Zoran Sodnik, and Nikos Karafolas
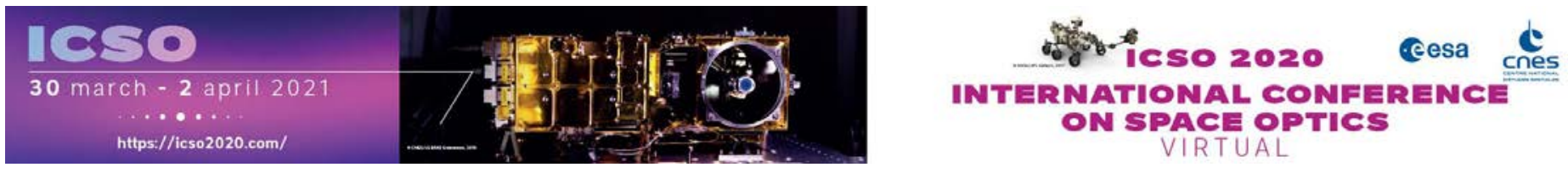

\section{Modular adaptive optics solution for a QKD receiver on a fork mount telescope system}

\section{Cesa issopoceatings lecnes}




\title{
Modular Adaptive Optics Solution for a QKD Receiver on a Fork Mount Telescope System
}

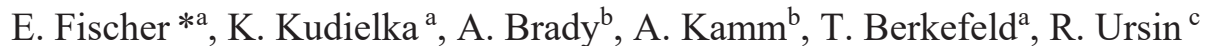 \\ ${ }^{a}$ Synopta GmbH, Eggersriet, Switzerland; ${ }^{b}$ Fraunhofer IOF, Jena, Germany; \\ 'IQOQI, Austrian Academy of Sciences, Vienna, Austria
}

\begin{abstract}
Quantum key distribution (QKD) is a promising field within telecommunication and cryptography. QKD allows two parties to generate a shared secret key, which only the two communicating parties know, and can later be used to encrypt and decrypt messages. This leads to unbreakable data transmission. QKD was experimentally already tested on a Chinese mission called MICIUS. Future QKD protocols may also benefit from coupling into an optical single-mode fiber. Adaptive optics is, from a quantum communication perspective, very interesting.

In this paper, we present the overall system design, the optical and mechanical design, and the factory performance test results, of an adaptive optics module for post-compensation of the quantum signal in a QKD ground station. The set-up is integrated in a compact and transportable "AO-box". The AO-box has been designed to be directly mounted on the flange of an $80 \mathrm{~cm}$ fork mount telescope and delivers the fiber-coupled quantum signal at its output interface.
\end{abstract}

Keywords: Quantum key distribution, QKD, adaptive optics, single-mode fiber

\section{INTRODUCTION}

In this paper, we present the overall system design, the optical and mechanical design, and the factory performance test results, of an adaptive optics (AO) module for post-compensation of the quantum signal in a QKD ground station. The set-up is integrated in a compact and transportable "AO-box". The AO-box has been designed to be directly mounted on the flange of an $80 \mathrm{~cm}$ fork mount telescope and delivers the fiber-coupled quantum signal at its output interface.

The optical design has prioritized high transmission and optical quality over a large bandwidth $\left(650 \mathrm{~nm}<\lambda_{\text {Beacon }}<\right.$ $730 \mathrm{~nm}, 750 \mathrm{~nm}<\lambda_{\text {Signal }}<860 \mathrm{~nm}$ ) as well as polarization insensitivity, allowing for flexibility in the design of future experiments, especially with respect to wavelength selection. The mechanical design has concentrated on providing a long-term robust system.

The dust-proof, closed AO-box is highly stable and thermally controlled, in order to maintain the optical quality in harsh temperatures and after transport. In addition, the AO-box includes an accessible "User-box" which, while isolated from the rest of the optical set-up, provides access to the fiber-coupling terminal and a mechanical interface for future optical elements.

*edgar.fischer@synopta.ch; www.synopta.ch

\section{SYSTEM AND SOFTWARE DESIGN}

The responsibility of the overall AO-box design, as well as the electrical and software engineering, final system integration, commissioning and performance testing of the AO-box fell to Synopta. The quantum communication AO- 
box developed for IQOQI is an upgrade to an existing Alt/Az mounted telescope. The Ritchey-Chrétien telescope has an entrance aperture of $80 \mathrm{~cm}$ and a f-number of 6 . The AO-box shall be mountable to the fork of the telescope mount at one of the two Nasmyth ports. Therefore, the AO-box is designed as vertically oriented optical bench that can be attached to the aforementioned fork of the telescope mount. The control and drive electronics for the AO-box is placed on the Nasmyth platform of the telescope and thus in the vicinity of the optical box.

In Figure 1 the top-level functional block diagram of AO-box interior is depicted. The box is equipped with an internal self-test source as well as with some further AO calibration sources. These sources allow a complete functional selftesting and also a radiometric calibration of the AO-system. When configured for nominal operation the downstream light, i.e. in receive direction, enters the box at a dust protection window. A fast tip/tilt mirror is used for line-of-sight stabilization. Therefore, the full stroke of the deformable mirror (DM) is available for correction of higher order aberrations in the received wavefront. The beacon camera shown in Figure 1 is primarily used as acquisition sensor. However, when the AO-loop is closed this camera can also be used as scoring (i.e. science) camera. A Shack-Hartmann sensor is used as wavefront sensor.

The optics of the AO-box is well corrected for a broad wavelength band that comprises the beacon and the QKD band (details see §3). For this reason, the shorter wavelength beacon light can be used to sense the received wavefront. The wavefront correction made by the AO system is in this case also correct for the faint light in the QKD band. A reflective dichroic filter is used to split off the QKD band from the beacon path. The QKD light is finally coupled into a singlemode fiber for further processing. A filter bench in the beacon path is equipped with optical neutral density filters. These filters can be moved into the beacon path in in cases where the dynamic range of the wavefront camera is not sufficiently large.

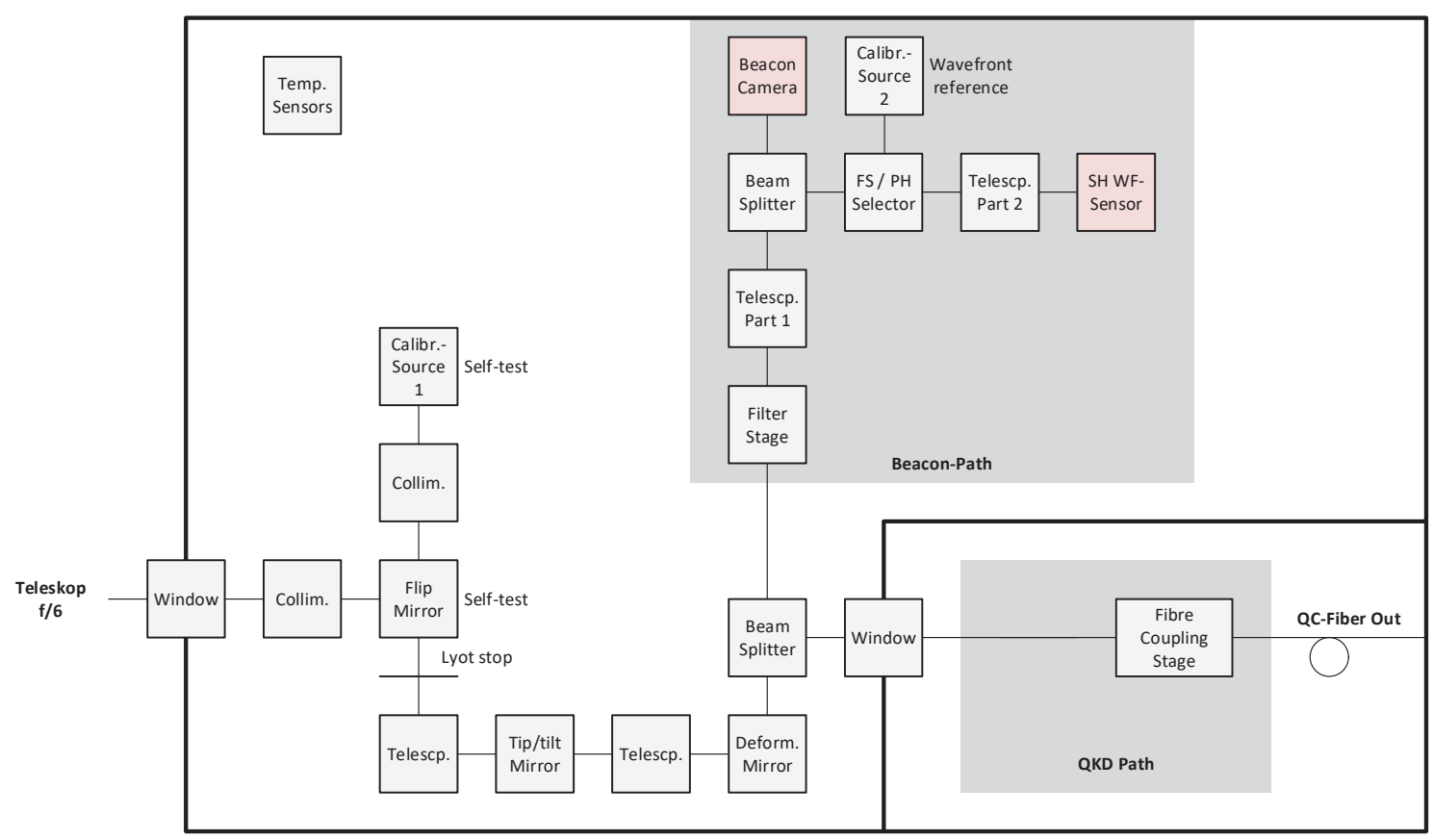

Figure 1. AO-box internal functional block diagram

The AO-software used in the AO-box is derived from Synopta's AO-software used in conventional satellite-to-ground optical link applications [1]. The software is implemented as server/client application. The user can therefore operate the system also remotely via network access or even via internet. In Figure 2 the main user interface is shown when running the AO. 


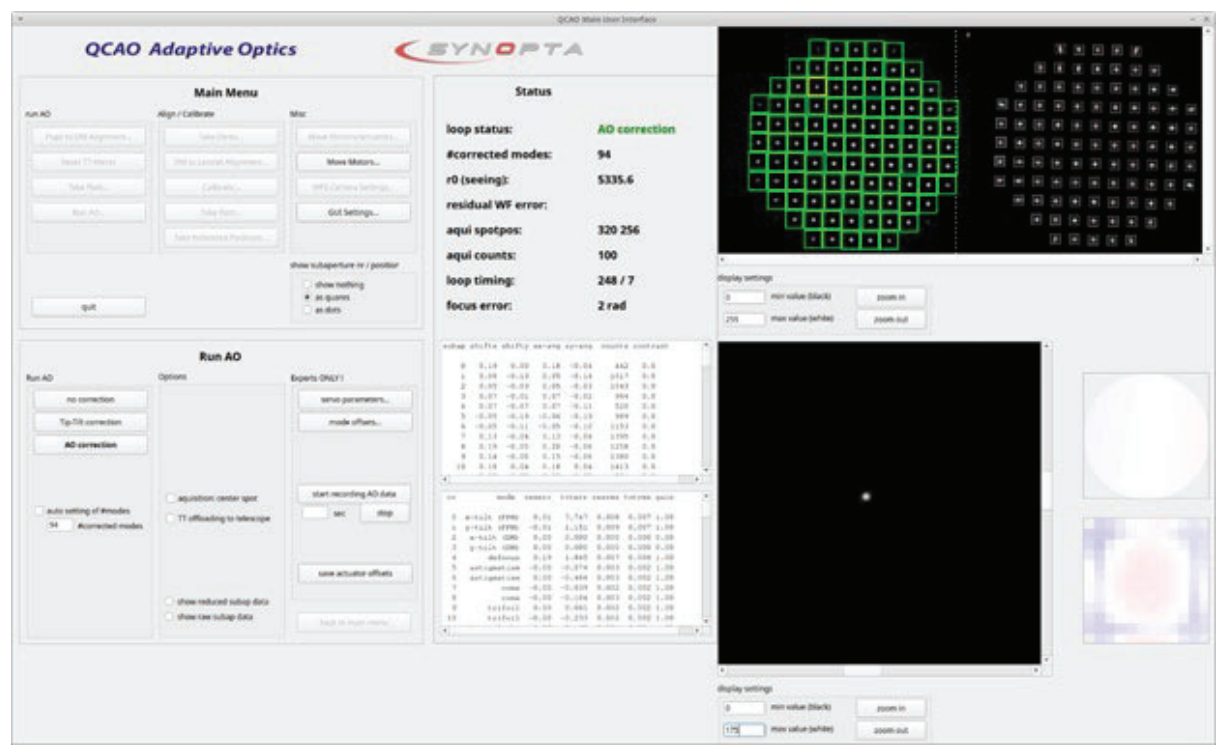

Figure 2. Main user interface, with "Run AO" menu.

\section{OPTICAL AND MECHANICAL DESIGN}

The responsibility of the optical and mechanical design of the AO-box, as well as the manufacturing and assembly and provisional testing of the passive system fell to Fraunhofer Institute for Applied Optics and Precision Engineering (IOF), Jena. The design goal of the AO-box was to provide a turbulence corrected quantum signal via single mode fiber output on the telescope. This meant that the system was installed at the Nasmyth focus and that the entire AO-system needed to be compact and light enough to fit on the telescope fork mount. The optical quality needed to be high enough to be suitable for quantum communication protocols and the alignment needed to be stable enough to be transportable and accurate long term. Moreover, the system needed to be closed and dust-proof as well as temperature controlled, in order to ensure reliable operation in wide ranging weather conditions. This put strict optical and mechanical requirements on the design.

The optic design of the QC-AO-box needed to have a high optical performance, while also meeting technical demands and restrictions to allow implementation of the system in a compact, transportable and modular system. Any channel loss needs to be accounted for in quantum communication protocols [2], and thus photon loss throughout the system needed to be minimized, enabling maximal single mode fiber coupling efficiencies. To ensure that all degrees of freedom provided by the adaptive optics could be used to correct for turbulence alone, the nominal optical performance of the system had to be optimized, so that even without adaptive optics and without turbulence effects, a desired Strehl ratio of $>90 \%$ in the quantum path could be achieved. Transmission was similarly critical, requiring values in excess of $80 \%$ across the full wavelength range, a challenge given the high number of passive optical elements required for phase conjugation in an adaptive optical system. Furthermore, given that quantum communication protocols often rely on polarization encoding of qubits [3], it was inherent that the design be polarization independent so as to prevent any unwanted "measurement" of the polarization states. Finally, in order to allow for flexibility in the choice of future experiments, it was important that the design be as broadband as possible. The design of the AO-box was therefore made for a quantum signal wavelength range of $750 \mathrm{~nm}<\lambda_{\text {Signal }}<860 \mathrm{~nm}$.

In order to allow operation of the adaptive optics loop independently of the quantum signal, an additional "beacon" signal was required. This allows correction of the weak quantum signal using wavefront measurements of the brighter beacon signal which enables rapid wavefront recording as an input to the adaptive optics control loop. The beacon signal was set at $685 \mathrm{~nm}$, with an extended wavelength range of $650 \mathrm{~nm}<\lambda_{\text {Beacon }}<730 \mathrm{~nm}$ for flexibility in compatibility with 
future optical systems. This resulted in the need for an achromatic design which fulfilled the stringent optical requirement over a range of $650 \mathrm{~nm}<\lambda<860 \mathrm{~nm}$.

With regards to the technical requirements - the AO-box needed to be compact and lightweight enough to be mounted directly on the telescope fork mount flange and be easily transportable, while also being robust enough to ensure a long term stable optical alignment. The system needed to be closed, dust-tight, and have the means to be thermally regulated. A separate telescope mount was required to provide both freedom of alignment and long-term stability on the telescope flange.

Adaptive optics systems require phase conjugation of the active planes of the system. The entrance pupil of the telescope needs to be conjugated to the planes of correction and that of wavefront measurement - in this case the tip-tilt mirror, deformable mirror and the wavefront sensor. This is performed with a series of Keplerian type telescopes [4]. This results in a large number of passive optical elements and a system with a very long optical path length. To limit the manufacturing costs of the system, it was decided to use only commercially available passive lenses. Small beam diameters and large F-number lenses were selected in the interests of ensuring optical quality over system path length, while nonetheless keeping the system as compact as possible. Mirrors enabled folding of the long optical path into a more compact form. The tip tilt and deformable mirrors were implemented under a small angle of $20^{\circ}$. This served to maintain full stroke of the mirrors and reduce ellipticity of the beam in the active planes. In addition to the tip tilt and deformable mirrors, further reflecting optics enabled folding of the optical path. Most had specific functionality, such as the first mirror in the optical system which serves as an alignment mirror between the telescope and AO-box, or the dichroic beam splitter which separated the quantum and beacon signals. Two additional folding mirrors were also included. Thus, the optical path could be sufficiently contained so as to enable implementation in an extremely compact modular unit.

Given the broadband nature of the system, achromatic lenses were required and given that transmission losses increase rapidly with successive passive elements, each element need a suitable broadband anti-reflection coating. For the mirrors, dielelectric coatings were selected for the mirror optics, as these provided both high reflectivity and minimal differences between the s- and p- polarization reflectance.

The foundation of the AO-box design was a monolithic aluminum breadboard of $20 \mathrm{~mm}$ thickness. The AO-box was then completed with further solid aluminum walls and a lid. Additional internal walls were used to enhance the stability. The breadboard flatness was measured to be under $100 \mu \mathrm{m}$ across its entire expanse. Low manufacturing tolerances allowed for a hybrid optomechanical concept, where commercial off-the-shelf lens and mirror mounts were combined with custom designed mounting posts using minimum degrees of freedom. Long term stability was prioritized over easeof-alignment or displacement range, so that very few spring-loaded mounts were implemented, and where unavoidable, only lockable elements were used.

Aside from the deformable mirror and tip tilt mirror, other electro-optomechanical elements were necessary, such as the customized ND filter stage, the two-axis motorized mirror which allowed alignment with the telescope, and the flip mirror for switching between the internal calibration source and the telescope input. Given the enclosed concept of the AO-box, these elements needed to be remotely operable. Electronic interface panels in the AO-box walls were therefore necessary to ensure the AO-box remained sealed and be transported separately from both the telescope and the surrounding electronics as necessary. Anti-reflection coated optical windows in the incoming and outgoing optical paths were included to ensure the dust proofing.

Thermal changes can lead to loss of alignment and thus a loss of performance, and therefore the AO-box needed a thermal regulation system and associated thermal isolation. A liquid heating and cooling concept was applied, which was integrated in the optical breadboard. While the closed and dust-proof concept of the box was considered essential to the long-term stable alignment of the AO system, access to the fiber coupling unit of the system was necessary. This was made possible through the inclusion of the so-called "User-box", as can be seen in Figure 3. This section of the AO-box was isolated from the rest of the box, with an optical window providing access to the optical signal and a separate lid. The User-box allows for flexibility in the choice of future experiment, providing direct access to the fiber coupling lens and unit of the system. The inclusion of additional optics as necessary is made possible through a "mini-breadboard", or grid of threaded bores which was implemented in the main breadboard. Thus, access to the end of the optical path is made possible, without compromising the isolation of the rest of the system. 

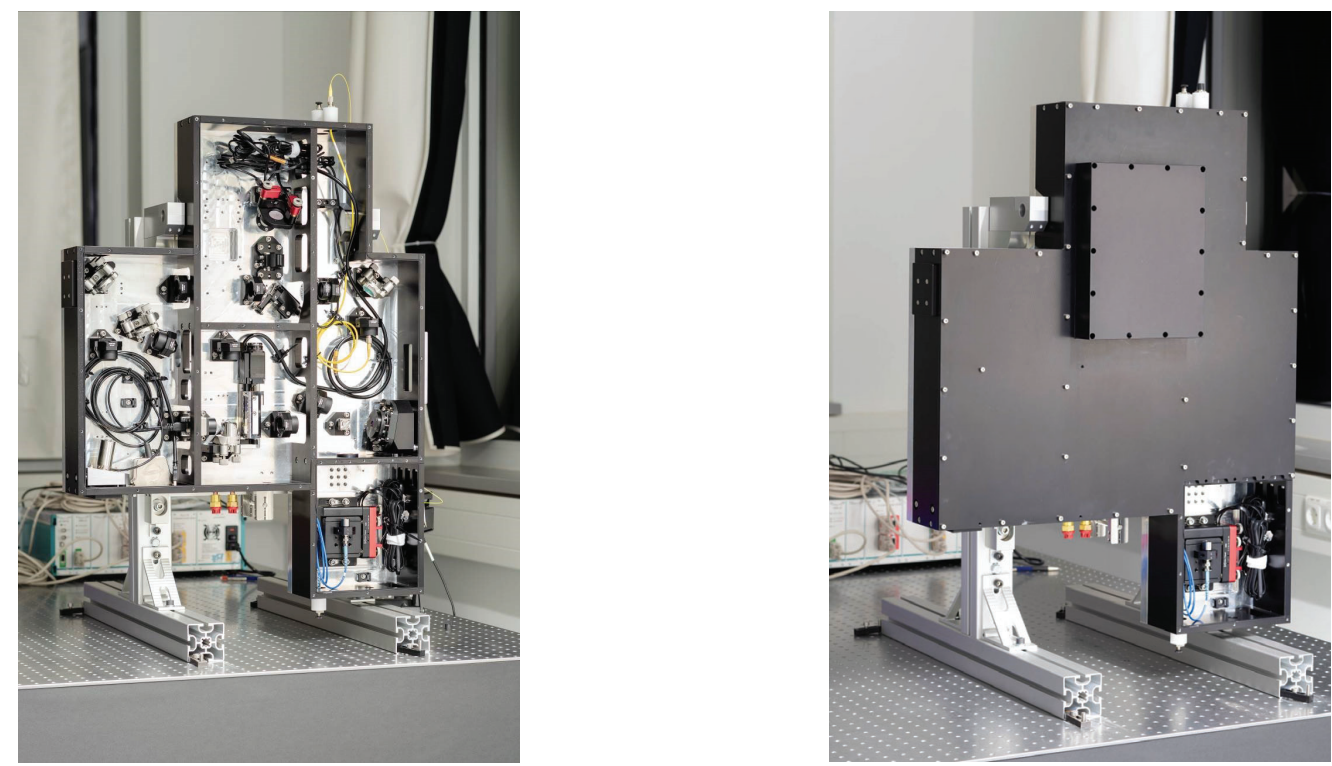

Figure 3. (Left) The AO-box after completion of all alignment and testing at Fraunhofer IOF. (Right) With the main lid installed, the accessibility of the User-box including the fiber-coupling unit and mini-breadboard is evident.

In order to allow for stable integration of the AO-box on the telescope mount, a hanging concept was implemented, whereby a forked mount was attached to the telescope flange and the AO-box itself hangs in this fork. This is to ensure stable positioning of the AO-box as it hangs in its center of gravity. Alignment was similarly performed in a vertical position. A sketch of the AO-box, as it will be installed on the telescope can be seen in Figure 4.

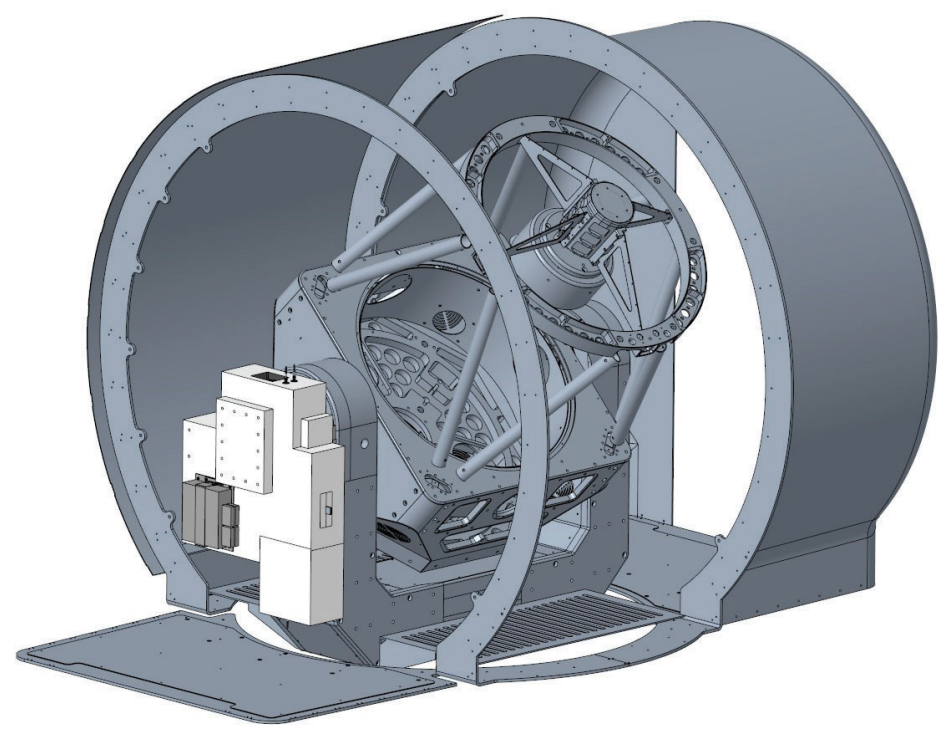

Figure 4. A construction model of the AO-box, as it will be installed on the telescope fork mount.

Following manufacturing and alignment, initial tests were performed at Fraunhofer IOF in order to evaluate the nominal optical quality of the system. The final system measured $830 \times 795 \times 250 \mathrm{~mm}$ prior to installation of the thermal isolation and weighed under $70 \mathrm{~kg}$. The Strehl ratio as measured with a wavefront sensor was measured to be $90.3 \%$ in the quantum path and $95.7 \%$ in the beacon path, fulfilling the design requirements for the nominal system. The measured 
transmission was negatively impacted due to the use of a substitute optical element for the deformable mirror, made necessary by the staggered AO integration. It measured $52.8+/-0.4 \%$, however for the path excluding this optical element, it was measured to be $89.0+/-0.8 \%$. Stability testing - whereby the optical performance was measured before and after a substitute transport, showed no change in alignment or optical quality. The optical performance was therefore consistent with the design specifications at the outset and the specially developed mechanical design was proven to provide sufficient stability for the optical setup. The optical and mechanical design of the system was therefore considered a success.

\section{PERFORMANCE TESTING}

Figure 5 shows the test setup used for the factory acceptance test. Light from two laser diodes (one at the beacon wavelength, one at the quantum communication wavelength) were combined by a wideband fiber-optic coupler. The laser diodes could be switched on or off individually. One coupler output was used to feed the beacon signal to the AObox internal self-test collimator. The second coupler output was used to feed the beacon and quantum communication signal into the AO-box (individually or simultaneously), via collimator, turbulence simulator, and telescope simulator (fnumber similar to that of the target telescope). A rotating phase plate was used as optical turbulence generator. Varying cross-wind speeds were simulated via different rotational speeds of the phase plate.

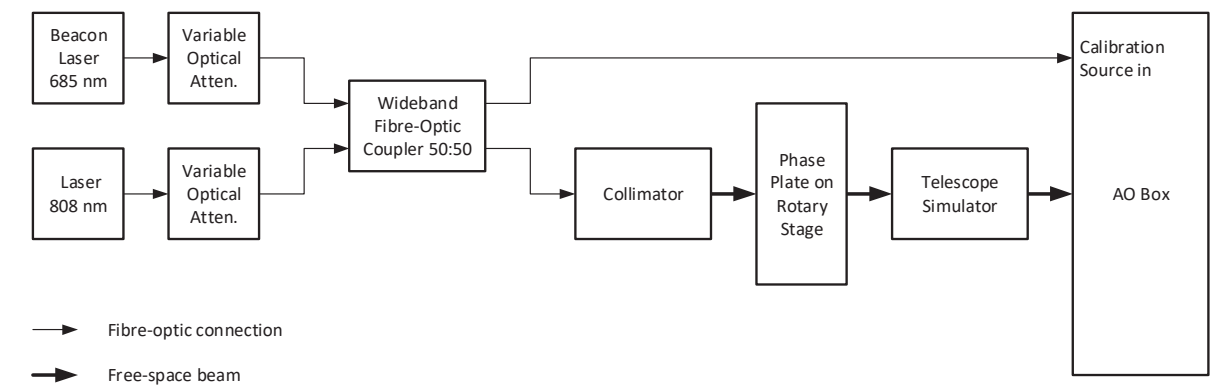

Figure 5. Test setup for factory acceptance test.

The factory acceptance test was successfully performed close to the minimum specified Fried parameter, namely $\mathrm{r}_{0}=37$ (a) $500 \mathrm{~nm}$, at different cross-wind speeds. The test results are summarized in Table 1 below.

The variation of the coupling efficiency between the ideal case (no turbulence) and the close-to-worst case (max. wind speed, $2 \mathrm{kHz}$ loop update rate) is very benign (Strehl ratio: approx. 0.52). This very good result could be achieved, thanks to the sufficiently large $r_{0}$ at $808 \mathrm{~nm}$ (only slightly below sub-aperture size), the low readout noise of the WFS camera, and the high closed-loop rejection cut-off frequency of the AO loop.

In addition to the fiber coupling test with simulated turbulence at nominal operational temperature a second test sequence was successfully performed that simulated also the maximum specified temperature difference between outside environment and box internal environment. In Figure 6 the AO-box is shown during the temperature test on the left-hand side of the photograph. The electronics units, necessary to operate the AO-box, are depicted in Figure 6 on the right-hand side. The system is ready for integration at the telescope. 
Table 1. Fiber coupling efficiency during factory acceptance test for no turbulence, or $\mathrm{r}_{0} @ 500 \mathrm{~nm}=37 \mathrm{~mm}$ and various wind speeds.

\begin{tabular}{|l|l|l|}
\hline AO Loop Update Rate & \multicolumn{1}{|c|}{ Efficiency (Lyot stop into fiber) } & \multicolumn{1}{c|}{ Remark } \\
\hline $2 \mathrm{kHz}$ & 0.54 & No turbulence \\
\hline $2 \mathrm{kHz}$ & 0.30 & $\mathrm{v}_{\text {wind }}=6.6 \mathrm{~m} / \mathrm{s}$ \\
\hline $2 \mathrm{kHz}$ & 0.28 & $\mathrm{~V}_{\text {wind }}=13.2 \mathrm{~m} / \mathrm{s}$ \\
\hline $4 \mathrm{kHz}$ & 0.54 & No turbulence \\
\hline $4 \mathrm{kHz}$ & 0.30 & $\mathrm{~V}_{\text {wind }}=6.6 \mathrm{~m} / \mathrm{s}$ \\
\hline $4 \mathrm{kHz}$ & 0.30 & $\mathrm{v}_{\text {wind }}=13.2 \mathrm{~m} / \mathrm{s}$ \\
\hline
\end{tabular}

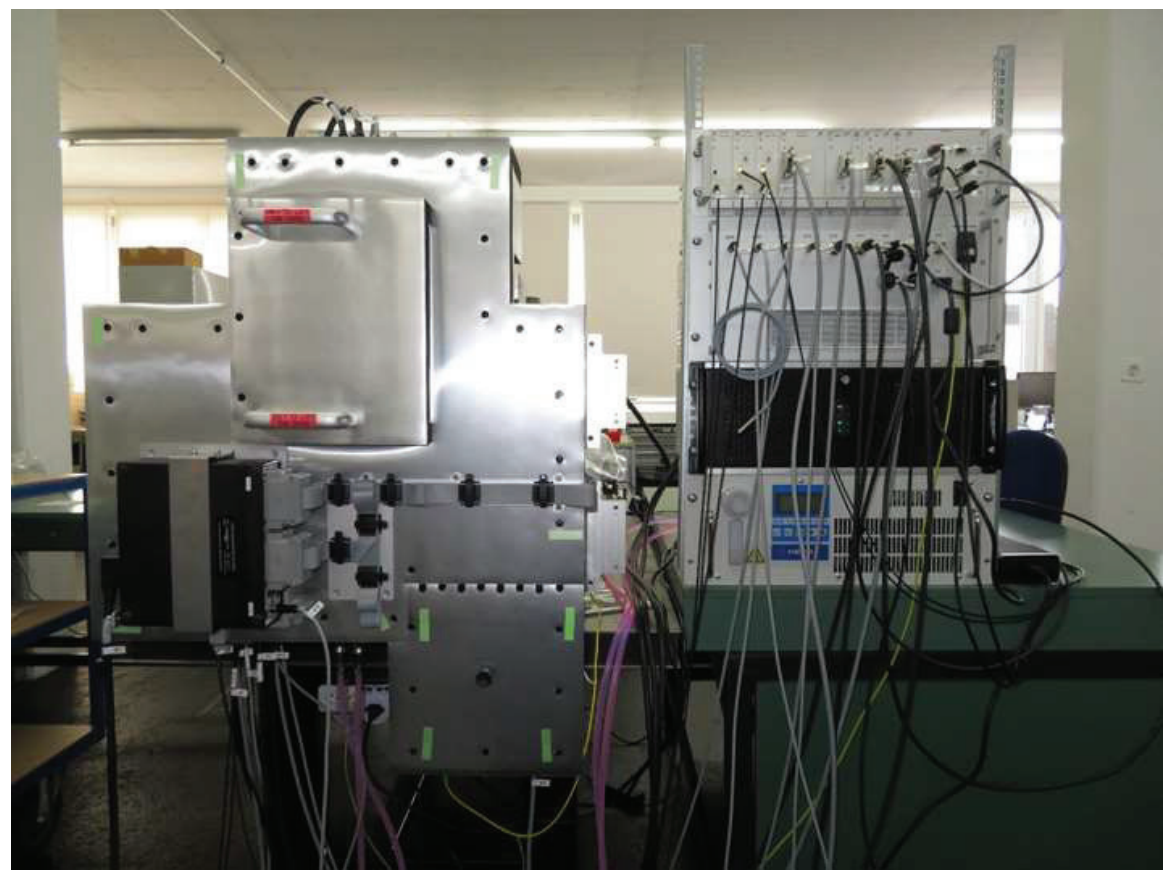

Figure 6. Photograph of AO Box and electronics during functional and thermal test.

\section{REFERENCES}

[1] Fischer E. et al., "Development, Integration and Test of a Transportable Adaptive Optical Ground Station", 2015 IEEE International Conference on Space Optical Systems and Applications (ICSOS), DOI: 10.1109/ICSOS.2015.7425071

[2] C. H. Bennett, G. Brassard, in Proceedings of IEEE International Conference on Computers, Systems, and Signal Processing, Bangalore, India (IEEE, New York, 1984), p. 175.

[3] Ecker, S., Liu, B., Handsteiner, J. et al. Strategies for achieving high key rates in satellite-based QKD. npj Quantum Inf 7, 5 (2021). https://doi.org/10.1038/s41534-020-00335-5

[4] Tyson, R., [Principles of Adaptive Optics], CRC Press (2010). 